\title{
Flow rate boundary conditions and multiscale modelling of the cardiovascular system in compliant domains
}

\author{
A. Moura \& C. Vergara \\ MOX (Modeling and Scientific Computing) Department of Mathematics, \\ Politecnico di Milano, Italy
}

\begin{abstract}
The prescription of boundary conditions is a critical issue in 3D blood flow simulations. To account for the whole cardiovascular system, we can impose data measurements or couple the $3 \mathrm{D}$ model with reduced ones (see $[4,5,9])$. In either cases only average (mean pressure or flow rate) data are normally available, which must be properly fed to the Navier-Stokes solver. In this paper we propose an algorithm to impose mean flow rate boundary conditions in a problem posed on a compliant domain. It is an extension of the approach advocated in [3, 12]. Furthermore we consider the coupling between a 3D fluid-structure interaction model and a lumped parameters (0D) one, representing the systemic tree. We extend the two coupling techniques in $[2,11]$. In the first approach the reduced model provides the mean pressure to be imposed as defective boundary condition to the 3D model, which conversely will make ready the flow rate to the reduced model. In the second strategy the type of data to be exchanged is reversed.
\end{abstract}

\section{Introduction}

Blood flow simulations often require a detailed description of the flow in a specific sub-region of the cardiovascular system (e.g. carotid bifurcation, stenosed vessel, etc.). These detailed information is obtained by means of three-dimensional (3D) fluid-structure interaction models based on the coupling of the incompressible Navier-Stokes equations (when considering medium to large vessels) with a structure model for the vessel wall. A particular vascular district of interest is considered, thus specific data on the artificial boundary sections (i.e. the part of the 
boundary not corresponding to the physical wall) have to be prescribed. Since the human cardiovascular system is formed by a closed network of vessels with a high level of interdependence, a realistic numerical simulation of the blood flow in a local district may not be fully accomplished if the interactions with the systemic haemodynamics are neglected. One possible solution is to resort to experimental measurements. Another possibility is to couple the 3D fluid-structure interaction model with reduced models of the cardiovascular system (see [4, 5, 9]). The latter leads to the geometrical multiscale modeling of the circulatory system, which consists in approximating the different parts of the cardiovascular system with different levels of accuracy, leading to different levels of computational complexity. The reduced models are derived from the 3D fluid-structure interaction model, by making some assumptions and simplifications (see [4, 5]). Either measurements and reduced models provide average data (mean pressure and flow rate). However, the 3D fluid-structure problem requires pointwise boundary conditions. So, in order to have a well posed problem, the non-standard boundary conditions have to be properly set.

For what concerns the mean pressure problem (i.e. the prescription of the mean pressure on the artificial section), in this paper we apply to a compliant vessel the approach proposed in [6], that suggests to impose on the artificial section some natural (Neumann) conditions obtained from a suitable variational formulation. For the flow rate problem, we present an extension to the compliant case of the augmented formulation proposed in [3, 12].

Moreover, we extend the 3D-0D coupling proposed in [2, 9], by coupling a lumped parameters model (0D) of the circulatory system with a $3 \mathrm{D}$ fluid-structure interaction model of a compliant cylindrical vessel. The coupling is performed enforcing the continuity of the flow rate and of the mean pressure on the artificial interface sections (denoted by $\Gamma_{j}$ ). Furthermore, an algebraic pressure-area relation is assumed on the 0D model, making possible for the $0 \mathrm{D}$ model to provide information on the wall displacement.

We propose two different coupling strategies, to be used in the frame of an iterative procedure. In the first approach the network provides a mean pressure value as defective boundary condition on $\Gamma_{j}$, while the $3 \mathrm{D}$ model returns the flow rate through $\Gamma_{j}$ to the network. Alternatively, the reverse situation can be considered. In this case the proposed approach to impose the flux is applied.

Section 1 is devoted to the 3D fluid-structure interaction coupling algorithm and Section 2 to its extension to the flux problem. In section 3 we introduce the lumped parameters model and we discuss its coupling with the $3 \mathrm{D}$ fluid-structure interaction one. Finally, in section 4 we present some preliminary numerical results.

\section{The fluid-structure interaction problem}

To describe the behaviour of the blood in compliant domains, we couple the Navier-Stokes equations, modelling the incompressible Newtonian fluids (like blood in sufficiently large vessels), with a model for the structure. Regarding the 
fluid problem, we can not use in this case an Eulerian approach, as usually done in fluid mechanics (see, for example, [10]).

In fact, since no slip conditions are prescribed on the physical boundary $\Gamma_{t}^{w}$, we would like to use Lagrangian coordinates near the wall. In this way the velocity of the fluid and of the structure are equal on $\Gamma_{t}^{w}$. However, a fully Lagrangian approach is not suitable, since far from the wall we would like the points of the fluid domain not to move, so that the domain does not warp (see Figure 1, right).
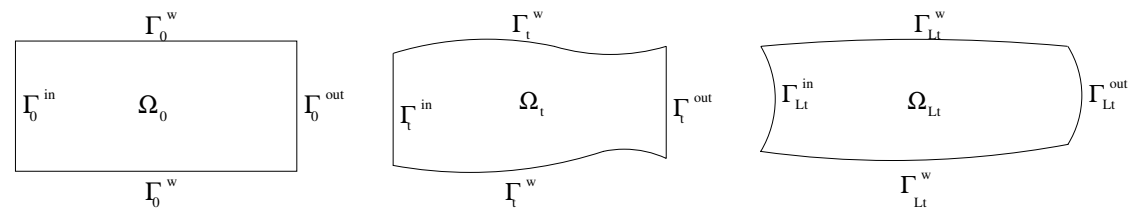

Figure 1: Reference (left), ALE (center) and Lagrangian (right) fluid domain.

In order to merge these requirements, let us introduce the Arbitrary Lagrangian Eulerian (ALE) approach (see [7, 8]). The idea is based on moving the points of the fluid domain in an arbitrary way, with the constraint that the points on $\Gamma_{t}^{w}$ move with the structure and the points on $\Gamma_{t}^{i n}$ and $\Gamma_{t}^{o u t}$ do not move. This can be made, for instance, by solving a Laplace equation (harmonic extension):

$$
\left\{\begin{array}{l}
-\triangle \boldsymbol{x}=\mathbf{0} \quad \text { in } \quad \Omega_{0} \\
\boldsymbol{x}=\boldsymbol{g} \quad \text { on } \quad \Gamma_{0}^{w} \\
x_{\boldsymbol{n}}=0 \quad \text { on } \quad\left(\Gamma_{0}^{\text {in }} \cup \Gamma_{0}^{\text {out }}\right) \\
\frac{\partial \boldsymbol{x}_{\boldsymbol{\tau}}}{\partial \boldsymbol{n}}=\mathbf{0} \quad \text { on } \quad\left(\Gamma_{0}^{\text {in }} \cup \Gamma_{0}^{\text {out }}\right)
\end{array}\right.
$$

being $\boldsymbol{x}$ the position of the points (whose normal and tangential components are $\boldsymbol{x}_{\boldsymbol{n}}$ and $\boldsymbol{x}_{\boldsymbol{\tau}}$, respectively) and $\boldsymbol{g}=\boldsymbol{x}_{0}+\boldsymbol{\eta}$, with $\boldsymbol{x}_{0}$ the position at $t=0$ and $\boldsymbol{\eta}$ the structure displacement. Problem given by (1) allows to build the domain $\Omega_{t}$, for all $t>0$ (Figure 1, center) and, at the discrete level, it provides the fluid mesh. Let us introduce the ALE mapping $A_{t}: \Omega_{0} \rightarrow \Omega_{t}$ giving, for each point $\boldsymbol{Y}$ of the reference domain $\Omega_{0}$, the position $\boldsymbol{y}$ at time $t$ of the node that at $t=0$ was in $\boldsymbol{Y}$, following not the trajectories of the fluid, but the arbitrary movement chosen (given, for example, by (1)), i.e. $A_{t}(\boldsymbol{Y})=\boldsymbol{y}(t, \boldsymbol{Y})$. Similarly to the Lagrangian case, the $A L E$ time derivative of a scalar quantity $f$ is given by $\frac{D^{A} f}{D T}=\frac{\partial f}{\partial t}+\boldsymbol{w}$. $\boldsymbol{\nabla} f$, where $\boldsymbol{w}(t, \boldsymbol{y})=\frac{\partial}{\partial t} \boldsymbol{y}\left(t, A_{t}^{-1}(\boldsymbol{y})\right)$ is the velocity of the fluid mesh. It is now possible to write the Navier-Stokes equations in $\Omega_{t}$ :

$$
\left\{\begin{array}{l}
\frac{D^{A} \boldsymbol{u}}{D t}-\operatorname{div} \nu\left(\nabla \boldsymbol{u}+\nabla^{t} \boldsymbol{u}\right)+[(\boldsymbol{u}-\boldsymbol{w}) \cdot \nabla] \boldsymbol{u}+\nabla p=\boldsymbol{f} \text { in } \Omega_{t} \\
\operatorname{div} \boldsymbol{u}=0 \text { in } \Omega_{t} .
\end{array}\right.
$$


Notice that for $\boldsymbol{w} \simeq \mathbf{0}$, i.e. far from the wall, we recover the Eulerian formulation, while for $\boldsymbol{w}=\boldsymbol{u}$, i.e. on the wall, we get the Lagrangian formulation. Problem given by (2) has to be completed with suitable initial and boundary conditions on $\Gamma_{t}^{\text {in }}$ and $\Gamma_{t}^{\text {out }}$.

For what concerns the structure, we consider the equations of linear elasticity:

$$
\frac{\partial^{2} \boldsymbol{\eta}}{\partial t^{2}}-\operatorname{div}\left(\sigma_{s}(\boldsymbol{\eta})\right)=\boldsymbol{h}
$$

being $\sigma_{s}$ the structure stress tensor and $\boldsymbol{h}$ a forcing term. To close the problem, we impose the interface conditions:

$$
\left\{\begin{array}{l}
\boldsymbol{u}(t, \boldsymbol{y})=\frac{\partial \boldsymbol{\eta}\left(t, A_{t}^{-1}(\boldsymbol{y})\right)}{\partial t} \quad \text { on } \Gamma_{t}^{w} \\
\boldsymbol{h}(t, \boldsymbol{Y})=\sigma_{f}\left(\boldsymbol{u}\left(t, A_{t}(\boldsymbol{Y})\right), p\left(t, A_{t}(\boldsymbol{Y})\right)\right) \boldsymbol{n} \quad \text { on } \quad \Gamma_{0}^{w}
\end{array}\right.
$$

where $\sigma_{f}$ is the fluid stress tensor (see [8]). In other words, the structure prescribes the velocity as Dirichlet boundary condition to the fluid and the latter gives the stress on the interface as forcing term to the structure.

\section{Flow rate boundary conditions}

We would like to impose on $\Gamma_{t}^{i n}$ the following flux condition:

$$
\int_{\Gamma_{t}^{i n}} \boldsymbol{u} \cdot \boldsymbol{n} d \sigma=Q(t)
$$

Navier-Stokes equations need pointwise conditions to be well posed. However, condition (3) gives only an average information on the surface. To fill this gap, we extend the augmented formulation proposed in $[3,12]$ to the compliant case. The main idea is to consider the flux condition as a constraint imposed through a Lagrange multiplier $\lambda$, so that the variational ALE formulation reads

$$
\left\{\begin{array}{l}
\left(\frac{D^{A} \boldsymbol{u}}{D t}, \boldsymbol{v}\right)+\nu\left(\nabla \boldsymbol{u}+\nabla^{t} \boldsymbol{u}, \boldsymbol{v}\right)+(((\boldsymbol{u}-\boldsymbol{w}) \cdot \nabla) \boldsymbol{u}, \boldsymbol{v}) \\
-(\operatorname{div} \boldsymbol{v}, p)+\lambda \int_{\Gamma_{t}^{i n}} \boldsymbol{v} \cdot \boldsymbol{n} d \sigma=(\boldsymbol{f}, \boldsymbol{v}) \\
(\operatorname{div} \boldsymbol{u}, q)=0 \\
\int_{\Gamma_{t}^{i n}} \boldsymbol{u} \cdot \boldsymbol{n} d \sigma=Q(t),
\end{array}\right.
$$

for all $\boldsymbol{v} \in \boldsymbol{V}$ and $q \in Q$, for suitable spaces $\boldsymbol{V}$ and $Q$ and where $(\cdot, \cdot)$ is the inner product in $L^{2}$. 
For the numerical solution of the fluid-structure interaction problem with a flow rate boundary condition (3), we merge the approaches proposed in $[1,12]$. Let us write the fluid and the structure problems in the following compact form:

$$
\left\{\begin{array} { l } 
{ u _ { \lambda } ^ { f } = ( \boldsymbol { u } , p , \lambda ) = \mathcal { F } _ { \lambda } ( u ^ { s } ) } \\
{ u ^ { s } = \boldsymbol { \eta } = \mathcal { S } ( u _ { \lambda } ^ { f } ) , }
\end{array} \quad \text { or } \quad \left\{\begin{array}{l}
u^{f}=(\boldsymbol{u}, p)=\mathcal{F}\left(u^{s}\right) \\
u^{s}=\boldsymbol{\eta}=\mathcal{S}\left(u^{f}\right)
\end{array}\right.\right.
$$

The first problem adopts (4) for the fluid solution while the second employs an ALE Navier-Stokes with usual boundary conditions (in the sequel we refer to this problem as classical). Both problems could be written as a fixed point equation on $u_{s}$ :

$$
u^{s}=\mathcal{S}\left(\mathcal{F}_{\lambda}\left(u^{s}\right)\right), \text { or } u^{s}=\mathcal{S}\left(\mathcal{F}\left(u^{s}\right)\right),
$$

respectively. In [1] a Newton algorithm is proposed to solve the classical fluidstructure interaction problem. Extending this approach to the first problem in (5), we obtain the following algorithm:

\section{For each time step \\ Do until convergence ( $n_{N}$ iterations)}

- (a) Given $u^{s}$, solve the fluid problem $u_{\lambda}^{f}=\mathcal{F}_{\lambda}\left(u^{s}\right)$

- (b) Solve the structure problem $\hat{u}^{s}=\mathcal{S}\left(u_{\lambda}^{f}\right)$

- (c) Evaluate the residual $\mathcal{R}=\left(u^{s}-\hat{u}^{s}\right)$

- (d) Solve the tangent problem $\left[D_{u^{s}} \mathcal{R}\left(u^{s}\right)\right] \delta u^{s}=-\mathcal{R}\left(u^{s}\right)$, i.e. do until convergence ( $n_{T}$ iterations)

- (d1) Solve the fluid problem $u^{f, k}=\mathcal{F}\left(\delta u^{s, k}\right)$

- (d2) Solve the structure problem $\delta \hat{u}^{s, k}=\mathcal{S}\left(u^{f, k}\right)$

- (e) Update rule: $u^{s} \longleftarrow u^{s}+\delta u^{s}$

where $D_{u^{s}}$ is the Frechet derivative operator, with respect to $u^{s}$. Notice that point (d) is carried out with an inner Newton algorithm, whose $k^{\text {th }}$ iteration consists on solving a classical fluid-structure problem. To solve the problem at point (a) we use the algorithm proposed in [12] (GMRes + Schur complement), whose computational cost is equal to 2 classical Navier-Stokes problems. Hence, the computational cost of the $j^{\text {th }}$ (external) Newton iteration is equal to $2+n_{T}$ classical Navier-Stokes problems, against the $1+n_{T}$ required in the classical case.

Remark Suppose we have $n+1$ artificial sections $\Gamma_{i}, i=0, \ldots, n$, where we wish to impose the fluxes $F_{i}$. In the compliant case we have $\sum_{i=0}^{n} F_{i}=\int_{\Gamma_{w}} \boldsymbol{u}$. $\boldsymbol{n} d \sigma$. Thus, we can impose the fluxes on all $\Gamma_{i}$, contrary to the rigid case where, since $F_{0}=-\sum_{i=i}^{n} F_{i}$, we can not impose the flux on $\Gamma_{0}$.

\section{Coupling 3D fluid-structure interaction and lumped models}

In order to prescribe data on the artificial boundaries of the $3 \mathrm{D}$ fluid-structure interaction problem, we couple it with a lumped parameters description of the remaining part of the cardiovascular system. 
There are two kinds of reduced models that can be used to describe the human circulatory system at a low computational cost: the 1D and the lumped parameters (0D) ones. We will consider here the latter.

Starting from the 3D incompressible Navier-Stokes equations in a compliant straight cylindrical vessel, under certain assumptions and integrating over the cross sections, one can derive a $1 \mathrm{D}$ model, stated in terms of hyperbolic equations along the axial coordinate of the vessel.

The lumped parameters models can be derived starting from the $1 \mathrm{D}$ model, by integrating the equations in space, and are expressed in terms of a system of ordinary differential equations (ODE). They describe the variation in time of the averaged pressure and flow rate in a specific terminal compartment of the circulatory system. Since they do not account for variations in space, they are often called zero-dimensional (OD) models.

In general, a lumped parameters model describing the whole circulatory system can be written as (see [5, 9])

$$
\left\{\begin{aligned}
\frac{d \mathbf{y}}{d t} & =\mathbf{A y}+\mathbf{b}+\mathbf{r} & , & t>0 \\
\mathbf{y} & =\mathbf{y}_{\mathbf{0}} & , & t=0
\end{aligned}\right.
$$

where $\mathbf{y}$ is the vector of the state variables, consisting of the mean pressure and flow rate in specific compartments, $\mathbf{A}$ is the system matrix, $\mathbf{b}$ derives from the $3 \mathrm{D}$ model boundary conditions, $\mathbf{r}(t)$ accounts for the heart action and $\mathbf{y}_{0}$ is the given initial datum. The lumped parameters model can be regarded as electric networks. Indeed, the flow rate can be seen as the electric current and the mean pressure as the voltage.

We assume that system (6) describes the entire (or a part) circulatory system and we substitute a part of that network with its correspondent 3D description. In an iterative frame to the solution of the coupled problem, the 3D fluid-structure interaction model provides pointwise information on the velocity and pressure which could be integrated to obtain the average data to be prescribed to the OD model. For each interface section $\Gamma_{j}$ there are two choices:

- The 3D model provides the flow rate $Q_{j}$ to the network while the latter computes the mean pressure $P_{j}$ to be input to the 3D model. In this case we have a mean pressure boundary condition for the Navier-Stokes equations on the interface boundary. To this aim, the approach proposed in [6] can be applied. Therein, we pose $\left.(-p \mathbf{n}+\nu(\nabla \mathbf{u}) \mathbf{n})\right|_{\Gamma_{j}}=-P_{j} \mathbf{n}$ as boundary condition for the Navier-Stokes problem on $\Gamma_{j}$. Regarding the $3 \mathrm{D}$ structure problem, since the $0 \mathrm{D}$ does not provide a priori any information on the wall displacement, we exploit an algebraic pressure-area relation valid for the lumped parameters model $([4,5])$ :

$$
P=\beta \frac{\sqrt{A}-\sqrt{A_{0}}}{A_{0}} \quad \text { with } \quad \beta=\frac{\sqrt{\pi} h_{0} E}{1-\xi^{2}},
$$

where $A$ denotes the area and $A_{0}$ the value of the artery area at rest; $h_{0}$ is the wall thickness at rest, $E$ is the wall Young modulus and $\xi$ the Poisson ratio. 
Thus, through relation (7), the $0 \mathrm{D}$ model supplies in this case also the area $A$ to the $3 \mathrm{D}$ model that is prescribed to the structure equation as a Dirichlet condition, supposing that the interface section $\Gamma_{j}$ is cylindrical and moves only radially.

- The 3D model provides the mean pressure $P_{j}$ to the network while the latter gives the flow rate $Q_{j}$ to the 3D model. In this case the flux problem for the Navier Stokes equations can be solved with the strategy of Section 2. Here the lumped model does not provide any value for the area, since no relation is available on the 0D model between flux and area, so on the structure an homogeneous Neumann boundary condition is imposed.

The numerical treatment of the coupling between the lumped parameters model and the $3 \mathrm{D}$ one is based on a splitting strategy (see [2,9]): at each time step each model provides the necessary data to the other one. The explicit forward Euler method is sufficient to solve the ODE system corresponding to the network (see [9]). This causes the computation of the coupled $0 \mathrm{D}$ and $3 \mathrm{D}$ discretized models to be completely separated at each time step (see $[2,9]$ ).

\section{Numerical results}

The first results we propose concern a simulation where the physiological flux of Figure 2 is imposed in a cylindrical compliant vessel. The flux corresponds to measurements and is imposed by means of the algorithm described in Section 2. We have considered the stiffness of the vessel wall sufficiently rigid in order to

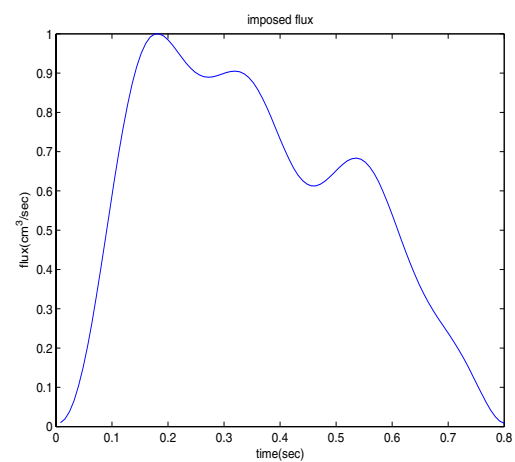

Figure 2: Physiological flux.

compare the results with the rigid case. It is evident from Figure 3 that the differences are negligible. We refer to future works for comparisons in more realistic cases. Since the experimental evidence showed that the number of inner Newton iterations $n_{T}$ is such that $5<n_{T}<9$, we conclude that the computational costs of the flow rate and classical problems are comparable for a cylindric compliant domain (see Section 2). 

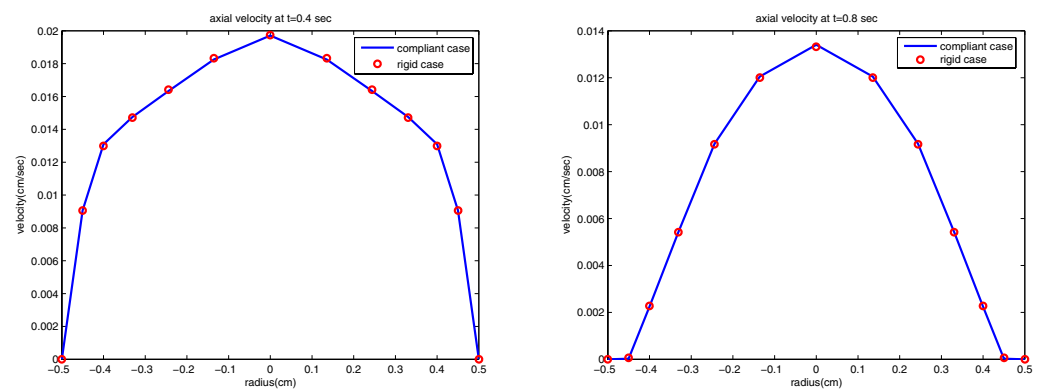

Figure 3: Axial velocity at the middle (left) and at the end (right) of the cycle.

Table 1: Comparative results for the 3D-0D coupling at the end of the cycle, using the strategy of imposing the mean pressure on the 3D model.

\begin{tabular}{|c|c|c|c|}
\hline & Coupling 3D-0D & Equivalent network & relative error \\
\hline$\Delta P$ & 0.6108 & 0.6074 & 0.0056 \\
\hline$Q_{\text {in }}$ & $5.675 \times 10^{-3}$ & $6.484 \times 10^{-3}$ & 0.1248 \\
\hline$Q_{\text {out }}$ & $5.726 \times 10^{-3}$ & $6.789 \times 10^{-3}$ & 0.1566 \\
\hline
\end{tabular}

The second simulation concerns the coupling of a 3D compliant cylinder with the simple network of Figure $4([2,12])$, which has a pressure source of type $\mathbf{U}(t)=c+\cos (2 \pi t)$, capturing the periodic action of the heart.

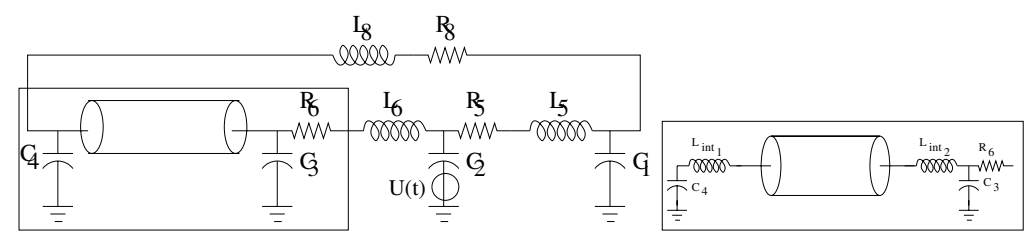

Figure 4: Network for the mean pressure problem (left) and its modification for the flux problem (right).

In Table 1 we present the results obtained for the coupling, comparing them with the results obtained by substituting the $3 \mathrm{D}$ model with its equivalent lumped parameters description. The results show that the mean pressure and the flow rate are the almost the same at the interfaces, in particular we recover very good results for the pressure. Notice that for the flux strategy problem we have to allocate inductances on the coupling interfaces (see $[2,11]$ ). 


\section{Acknowledgments}

The authors wish to thank L. Formaggia and A. Veneziani for their relevant suggestions. The first author has been supported by European Union under contract HPRN-CT-2002-002670. The numerical solutions obtained were developed in $\mathrm{C++}$ in the scope of the finite element library $\mathrm{LifeV}$ with the relevant help of C. Prud'homme (www.lifev.org).

\section{References}

[1] Fernández, M. \& Moubachir, M., A Newton method using exact jacobians for solving fluid-structure coupling. Computers \& Structures, 83(2-3), pp.127$142,2005$.

[2] Fernández, M., Moura, A. \& Vergara, C., Defective boundary conditions applied to multiscale analysis of blood flow. ESAIM: CEMRACS Proceedings, 2005. Submitted.

[3] Formaggia, L., Gerbeau, J.F., Nobile, F. \& Quarteroni, A., Numerical treatment of defective boundary conditions for the Navier-Stokes equations. SIAM J Num Anal, 40(1), pp. 376-401, 2001.

[4] Formaggia, L., Nobile, F., Quarteroni, A. \& Veneziani, A., Multiscale modeling of the circulatory system: a preliminary analysis. Computing and Visualizations in Science, 2, pp. 75-83, 1999.

[5] Formaggia, L. \& Veneziani, A., Reduced and multiscale models for the human cardiovascular system. Lecture notes VKI Lecture Series 2003-07, Brussels.

[6] Heywood, J., Rannacher, R. \& Turek, S., Artificial boundaries and flux and pressure conditions for the incompressible Navier-Stokes equations. Int $J$ Num Meth Fluids, 22, pp. 325-352, 1996.

[7] Nobile, F., Numerical Approximation of Fluid-Structure Interaction Problems with Application to Haemodynamics. Ph.D. thesis, EPFL, 2001.

[8] Quarteroni, A., \& Formaggia, L., Modelling of Living System, Elsevier, chapter Mathematical Modeling and Numerical Simulation of the Cardiovascular System. Handbook of Numerical Analisys Series.

[9] Quarteroni, A., Ragni, S.\& Veneziani, A., Coupling between lumped and distributed models for blood flow problems. Computing and Visualizations in Science, 4, pp. 111-124, 2001.

[10] Quarteroni, A., \& Valli, A., Numerical Approximation of Partial Differential Equations. Springer, 1994.

[11] Quarteroni, A., \& Veneziani, A., Analysis of a geometrical multiscale model based on the coupling of ODEs and PDEs for blood flow simulations. Multiscale Model Simul, 1(2), pp. 173-195, 2003.

[12] Veneziani, A. \& Vergara, C., Flow rate defective boundary conditions in haemodynamics simulations. Int J Num Meth Fluids. 47, pp. 803-816, 2005. 